Andrea Zink, Ulrich Schmid

\title{
Theorien des Romans: Lukács und Bachtin
}

\section{Voraussetzungen}

Erst seit den achtziger Jahren zieht die Gegenüberstellung der romantheoretischen Positionen von Lukács und Bachtin das Interesse der Literaturwissenschaft auf sich (Kovács 1980, Aucouturier 1983, Corredor 1983, Wegner 1987, Neubauer 1996, Tihanov 1997a, 1997b, 1997c, 1998a, 1998b). Dabei liegt das Thema nahe: Es darf als gesichert gelten, daß Bachtin Lukács” „Theorie des Romans“ in den frühen zwanziger Jahren gelesen hat (Clark; Holquist 1984, 99). Außer der „Theorie des Romans“ kennt Bachtin Lukács' Artikel, die 1935 in der Zeitschrift „Literaturnyj kritik“" erschienen sind (Tihanov 1997c, 274). Grundsätzlich ist festzuhalten, daß zwischen den beiden Denkern nur ein einseitiges Rezeptionsverhältnis besteht. Lukács' Russischkenntnisse reichen auch während seiner Moskauer Zeit nicht dazu aus, anspruchsvollere theoretische Texte zur Kenntnis zu nehmen (ebd., 275). Außerdem wendet er sich in den zwanziger Jahren einer marxistischen Literaturkonzeption zu, die sich hauptsächlich für die künstlerische Widerspiegelung gesellschaftlicher Wirklichkeit interessiert.

Um so nachhaltiger wirkt aber Lukács auf Bachtin. In verschiedenen Phasen seiner theoretischen Entwicklung (Morson; Emerson 1990, 66) reagiert Bachtin zum Teil direkt auf Lukács, allerdings gelangt er bei einzelnen Fragestellungen nicht selten zu einer gegensätzlichen Einschätzung.

Schon in Lukács' und Bachtins frühen Schriften, die vor der expliziten Beschäftigung mit dem Roman entstehen, zeigen sich interessante Übereinstimmungen. Diese Arbeiten reflektieren die Krisenstimmung des beginnenden 20. Jahrhunderts und verfolgen ein ethisches Interesse. Von Anfang an distanzieren sich beide Denker von einer rein formalistischen Kunsttheorie, die technische Verfahren ohne Rücksicht auf ihre soziale Funktion untersucht. In der Tradition Kants situieren Lukács und Bachtin die Kunst zwischen Erkenntnis und Handeln; wie die Neukantianer gehen sie von einer Normorientierung des theoretischen, praktischen und ästhetischen Verhaltens aus. Die Kunst ist nicht wertfrei und soll es auch nicht sein. Durch die spezifische Art ihrer Normorientierung gleicht die Kunst allerdings mehr dem verantwortungsvollen Handeln als theoretischer Erkenntnis. Die Gnoseologie in Form der Logik kann für beide Denker zwar die allgemeingültigen Richtlinien der Erkenntnis benennen, den Bezug zu ihrer Erfüllung aber nicht herstellen (Lukács 1974, 92 und 111, Bachtin 1994a, 12f.). Die Norm bleibt dementsprechend abstrakt, an einem „Bewußtsein überhaupt" orientiert, ohne dem konkreten Bewußtsein einen Weg zu zeigen (Lukács 1974, 92, Bachtin 1994a, 15). ${ }^{1}$ Zwischen den theoretischen Regeln und dem wirklichen Denken der Menschen klafft sowohl für Lukács als auch für Bachtin ein Abgrund. Analoges aber gilt auch für die Ethik, von der man eine Orientierungsleistung erhofft hatte. Beide Denker konstatieren ein Ungenügen: Lukács sieht in der

Selbst noch in „Problemy tvorcestva Dostoevskogo“ polemisiert Bachtin gegen ein abstraktes „Bewußtsein überhaupt“. Der Begriff - im Original deutsch zitiert - entstammt der Philosophie Rickerts (1994b, 61f.). 
Ethik einen unüberwindbaren Abstand zwischen transzendentem Wert (der Freiheit des Subjekts) und konkreter Wirklichkeit (Lukács 1974, 93f.); ${ }^{2}$ Bachtin kritisiert die Abstraktheit ethischer Normen für die Menschen, die sie realisieren sollen (Bachtin 1994a, 14, 26ff.). Er entwirft ein Gegenmodell, das auf der fundamental verschiedenen Wahrnehmung des Ich von sich selbst und einem Anderen (dem Du) basiert. Menschliches Handeln ist immer durch diese doppelte Perspektivierung bedingt (ebd., 51f.). Die konkrete Tat hat gegenüber dem ,ästhetischen Sehen“ den Vorteil, daß sie sämtliche Bezugsmomente von Ich und Du in sich birgt, vor allem den Selbstbezug, in dem Bachtin die Verantwortung gegenüber dem eigenen Handeln lokalisiert. Das ,ästhetische Sehen“ erweist sich dagegen als nicht vollwertig. Der Schöpfer des Werks zieht sich nämlich aus der Tat zurück, er übernimmt nur begrenzt Verantwortung, weil er sein Produkt als selbständiges freigibt (21f.).

Bachtin und Lukács gemeinsam ist die Einschätzung, daß Gnoseologie und Ethik - zumindest eine „formale“ (Bachtin 1994a, 31) - durch die Unendlichkeit ihres Anspruches und die daraus folgende problematische praktische Umsetzung gekennzeichnet sind. Dies gilt jedoch nicht für die ästhetische Tätigkeit und ihr Objekt. Der Künstler setzt in seinem Werk bewußt eine Grenze, er schaltet Unendlichkeit aus, indem er ein Einzelnes, Konkretes und Abgeschlossenes thematisiert. In dieser Begrenzung besteht die wichtigste Leistung des Kunstwerks. Es kann, was Erkennen und Handeln gerade nicht können, nämlich im Immanenten über sich selbst hinausweisen. Lukács spricht von der Transzendenz im Immanenten (Lukács 1974, 113), Bachtin von der Transgredienz des dargestellten Lebens, die durch den Abschluß, die „Umarmung“ von seiten des Autors entsteht (Bachtin 1994a, 96ff., 141, 235, 238f.).

Das von Bachtin noch in „K filosofii postupka“ konstatierte Ungenügen des ästhetischen Sehens wird wenig später in „Avtor i geroj v ėstetičeskoj dejatel'nosti“ zusehends zum Vorteil umgedeutet. Der Künstler nimmt sein Objekt - und das sind für Bachtin vor allem Menschen - von außen wahr. Aber Distanz heißt nicht Indifferenz. Gerade die Außenposition ermöglicht dem Autor und seinem Rezipienten, sich in einen anderen Menschen „einzuleben“ (вживание), ohne sich jedoch mit ihm zu identifizieren. Hierin liegt der ästhetische Wert, der einen ethischen in sich birgt (ebd., 22, 24, 57f., 82, 89, 95ff.). Das Beispiel Ödipus, das sowohl Lukács als auch Bachtin zitieren, ist aufschlußreich (Lukács 1911, 30, Bachtin 1994a, 143ff.). Das Mitleid mit dem Helden - so Bachtin - entsteht erst durch die ästhetische Form. Eine entscheidende Rolle spielt dabei die olympische Perspektive, die der Autor mit dem Rezipienten teilt. Der Handelnde selbst empfindet nur Schrecken und sieht nichts als die Ausweglosigkeit seiner Situation. Die Tragik zeigt sich dagegen nur dem Betrachter, der außerhalb der Ereignisse steht. In ähnlicher Weise zieht Lukács bei Kierkegaard eine Trennlinie zwischen Leben und Dichtung. Kierkegaard wollte in seiner Beziehung zu Regine Ohlsen „leben, was man nicht leben kann“, „wollte Formen schaffen aus dem Leben“. Der unendliche Anspruch aber läßt jede konkrete Realisierung als ungenügend erscheinen, die Liebe würde zur Ehe versteinern. Ein Formen des Lebens gelingt nur dem Dichter (Lukács 1911, 88). Das künstlerische Gestalten weist eine

2 Dieses Problem unterlegt Lukács auch Kierkegaards gescheiterter Beziehung zu Regine Ohlsen (Lukács 1911, 61-90). 
ethische Dimension auf: „Eine richtende Kraft, ein Ethisches ist das Gestaltenkönnen und ein Werturteil ist in jedem Gestaltetsein enthalten." (ebd., 370)

Das gemeinsame Thema der frühen Ästhetik von Lukács und Bachtin - der Wert der Kunst für das Leben - zieht sich durch Schriften mit verschiedenster Thematik. Heterogen sind auch die literarischen Beispiele, an denen die Theorie dargestellt wird. Es gibt zunächst keine Präferenz für eine bestimmte Gattung. Erst in einer späteren Phase entdecken beide Denker den Roman als paradigmatischen Ort ihrer Ästhetik. Bachtin modifiziert dabei seine ursprüngliche Konzeption stärker als Lukács. ${ }^{3}$

Anhand eines thematischen Beispiels läßt sich dieser Wechsel besonders deutlich darstellen. Die Vollendung eines Kunstwerkes basiert - so Bachtin zu Beginn der zwanziger Jahre - auf der Außerhalbbefindlichkeit (вненаходимость) des Autors, auf seinem Überschuß an Wissen gegenüber dem Helden. Das Ereignis par excellence, das nur von außen beobachtet werden kann, ist der Tod (Bachtin 1994a, 169ff.). Deshalb steht für Bachtin nicht nur der Mensch, sondern besonders der sterbliche Mensch im Zentrum des ästhetischen Sehens $(60,71 \mathrm{f}$.). Kunst ist im Gegensatz zum ethisch bestimmten, unendlichen und offenen Handeln immer auf die Vergangenheit bezogen (ebd., 177). Dem formalen Kriterium der Vollendung entspricht auch die inhaltliche Ausrichtung auf Geschehenes. Später wird Bachtin allerdings von der Forderung nach Vollendung abrücken und das Gegenteil propagieren: In „Problemy tvorčestva Dostoevskogo" anerkennt er die Offenheit der Charaktere als epochale künstlerische Leistung Dostoevskijs.

Lukács' Konzeption weist in dieser Hinsicht Kontinuität auf. Seine Sehnsucht nach Vollendung, Totalität und dem kommenden Gott deutet sich in den frühen Schriften an (Lukács 1911, 35ff.) und setzt sich in der ,Theorie des Romans“ - wenngleich in einer anderen, geschichtsphilosophischen Argumentation - fort. Im Gegensatz zu Bachtin impliziert Lukács' Begriff der Vollendung aber keineswegs den Bezug auf Vergangenes.

Die folgende typologische Gegenüberstellung von Lukács' und Bachtins Romantheorien konzentriert sich auf drei Problemkreise. Erstens läßt sich ein auffallendes gemeinsames Interesse für das Werk Dostoevskijs feststellen. Zweitens spielt die Behandlung der Zeit bei beiden Romantheoretikern eine wichtige Rolle. Schließlich soll drittens Lukács' Ironieverständnis mit Bachtins Karnevalskonzept verglichen werden.

\section{Epopöe der Moderne vs. polyphoner Roman: Dostoevskij}

Die augenfälligste Übereinstimmung zwischen Lukács und Bachtin bietet ihr Interesse für das Romanschaffen Dostoevskijs. Ursprünglich konzipiert Lukács seine „Theorie des Romans" (1916) als Einleitung zu einem Dostoevskij-Buch, das allerdings nie ausgearbeitet wird und nur in vorbereitenden Notizen dokumentiert ist. Bachtin revidiert seine frühe

3 Der Einschätzung von Eva Corredor (1983, 107), derzufolge Lukács seine Ansichten immer wieder geändert habe, während Bachtins Denken annähernd konstant sei, ist deshalb nicht zuzustimmen. Michel Aucouturier postuliert ebenfalls einen direkten Zusammenhang zwischen Bachtins Frühwerk und seinem Dostoevskij-Buch. Das letztere exemplifiziere die Beziehung von Autor und Held, wie sie Bachtin in „Avtor i geroj" entworfen habe (Aucouturier 1983, 129). Daß hier ein Mißverstăndnis vorliegt, zeigt aber gerade Bachtins frühe Dostoevskij-Kritik: Dostoevskij hat nach Bachtin eine zu schwache Außenposition, er verliert sich an die Helden und kann die künstlerische Vollendung nur ungenügend realisieren (Bachtin 1994a, 252f.). Tihanov sieht in dieser Textstelle den Keim von Bachtins Dostoevskij-Buch, blendet aber die konträre Wertung aus (Tihanov 1997a, 189). 
theoretische Position anhand einer Dostoevskij-Interpretation, die er in seinem Buch „Problemy tvorčestva Dostoevskogo" (1929) vorlegt. Sowohl für Bachtin als auch für Lukács markieren Dostoevskijs Romane einen Wendepunkt in der Geschichte der Literatur. Allerdings rücken beide Interpreten je einen verschiedenen Aspekt von Dostoevskijs innovativer Kunst in den Blick: Lukács nimmt eine dialektische historische Entwicklung an, die vom Epos über den Roman zu Dostoevskijs Werken führt. Dostoevskij hat in Lukács' Sicht eine neue Form geschaffen, die - auf einer höheren Ebene - erneut Wesenszüge der Epopöe in sich aufnimmt. Auch Bachtin denkt historisch, jedoch weniger zielgerichtet. Er postuliert eine zweigleisige Entwicklung des Romans, wobei er eine monologische und eine dialogische Linie unterscheidet. Das Epos läßt er zunächst außer Acht. Bachtin attestiert Dostoevskij, die dialogische Entwicklungslinie zur Perfektion geführt zu haben: Dostoevskijs Leistung besteht in der konsequenten Gestaltung einer Vielzahl von menschlichen Standpunkten, von Stimmen, die selbständig und ohne Wertung durch den Autor zur Geltung kommen. Seine Romane sind polyphon. Damit gelangen Bachtin und Lukács zu entgegengesetzten Resultaten: Für Bachtin ist Dostoevskij ein Autor, der auf eine Synthese von Einzelpositionen verzichtet, Lukács hingegen billigt ihm gerade das Erreichen einer höheren Synthese zu.

Die unterschiedliche Deutung der Werke Dostoevskijs basiert mitunter auf einer überraschend ähnlichen Charakteristik. Übereinstimmend weisen Lukács und Bachtin darauf hin, was sich bei Dostoevskij nicht findet, nämlich das Problem des Todes. Diesen auffälligen Unterschied zu anderen Schriftstellern der Epoche führt Bachtin auf die untergeordnete Rolle zurück, welche die Geschichte - sowohl die individuelle als auch die gesellschaftliche - bei Dostoevskij spielt (Bachtin 1994b, 40). Seine Helden erscheinen nicht in verschiedenen Lebensabschnitten; nicht ihr Werden, sondern ihre Koexistenz und Wechselwirkung sind von Bedeutung (ebd., 39). Analog konstatiert Lukács in Dostoevskijs Werk die untergeordnete Rolle der biographischen Zeit, die kennzeichnend für den Roman ist (Lukács 1920, 70). Konsequenterweise gelangt Lukács deshalb zu dem Schluß, Dostoevskij habe „keine Romane geschrieben“ (ebd., 168).

Bachtin überträgt das ethische Konzept seiner frühen Schriften auf die Figuren des Romans. Die Besonderheit und Leistung des Autors Dostoevskij besteht darin, daß er seinen Geschöpfen einen Horizont (кругозор) (Bachtin 1994b, 78f.) zubilligt, mit dem sie sich gegen ideologische Fremddeterminierung (das Abschließen durch ihre Umgebung [окружение]) schützen. Sie kämpfen gegen fremde Meinungen über sich selbst und für die Unabschließbarkeit ihrer eigenen Persönlichkeit. Weder die gesellschaftliche Herkunft noch das biographische Ende, der Tod, können hier Bedeutung erlangen. Dostoevskij gestaltet das Nebeneinander gleichwertiger Persönlichkeiten. Deshalb dominiert die räumliche vor der zeitlichen Ordnung (ebd., 38). Der Verzicht des Autors auf eine abschließende Wertung läßt eine zeitlose Gemeinschaft selbständiger Seelen entstehen, für die Bachtin das Bild der Kirche verwendet, in der sowohl Sünder als auch Gerechte Platz finden (ebd., 37). Das Bild der Kirche will er aber explizit nur als Bild verstanden wissen, das keine metaphysische Deutung der Welt Dostoevskijs darstellen soll. Bachtin setzt den Akzent auf die Konstruktion; in „Problemy tvorčestva Dostoevskogo“ zeigt er sich zum ersten Mal als Literaturwissenschaftler. Seine Auseinandersetzung mit dem Formalismus hat dabei zweifellos Spuren hinterlassen, wenngleich Bachtin Dostoevskijs Pluralismus von 
Persönlichkeiten und Ideen nicht als wertfreie und damit auch wertlose Technik begreift. ${ }^{4}$ Der polyphone Roman hat historisch gesehen eine grundlegende Bedeutung. Es ist dafür aber die Polyphonie als Darstellungsprinzip verantwortlich, nicht die einzelnen Inhalte. Mit anderen Worten: Für Bachtin bleibt zweitrangig, welche Ideen Dostoevskijs Figuren vertreten; entscheidend ist die Vielfalt der ideologischen Positionen und ihre Bezogenheit auf ein persönliches Bewußtsein.

Nach Lukács' Dafürhalten transzendieren die Werke Dostoevskijs die Romanform. Mit dieser grundlegenden, jedoch nicht weiter ausgeführten These schließt die „Theorie des Romans“. Ihre ,Fortsetzung", die ursprünglich geplante Dostoevskij-Studie, liegt nur in Notizen vor. Obwohl eine Spannung besteht zwischen diesen Notizen und der „Theorie des Romans" (Lukács 1985, 13), ${ }^{5}$ läßt sich mit ihrer Hilfe doch die Charakterisierung Dostoevskijs präzisieren, die in der „Theorie des Romans" nur knapp und ex negativo vorgenommen wird. Was sind Dostoevskijs Werke, wenn sie „keine Romane“ sind? Zunächst einmal kann für Dostoevskijs Werk all das nicht gelten, was für den Roman und seine geschichtlichen Voraussetzungen zutrifft. Insbesondere muß hier eine andere transzendentale Bedingung vorliegen. Dostoevskijs Helden agieren nicht im Zeichen „transzendentaler Obdachlosigkeit“, sie sind keine Suchenden. Folglich steht hier auch kein Individuum mit seinen Idealen der strengen Welt der Konvention gegenüber. Der Kampf gegen die Konvention ist ein „Problem für Erwachsene“ (ebd., 133), ebenso wie der Roman die dichterische Form der „gereiften Männlichkeit“ (Lukács 1920, 63) darstellt. In Dostoevskijs Welt aber treten erneut „Kinder“ auf (Lukács 1985, 133), hier spielt die Zeit als eine Grundkategorie des Romans, die der Diskrepanz von Idee und Wirklichkeit Rechnung trägt (Lukács 1920, 127), keine Rolle. So attestiert auch Lukács - wie Bachtin - den Helden Dostoevskijs eine überzeitliche Verbundenheit (Lukács 1985, 28, 143, 181). Sie geht auf einen Zustand der Erlöstheit zurück, den die empirische Welt nur verschleiert. „Das Gottesreich ist in euch" - mit diesem Problem haben es Dostoevskijs Helden zu tun, nicht mit der Suche nach Gott (ebd., 70). Deshalb steht auch nicht die einsame, von Hoffnung getriebene Seele im Mittelpunkt, sondern die Möglichkeit einer Gemeinschaft. Die „transzendentale Obdachlosigkeit“ scheint sich in einen erneuten Schutz Gottes verändert zu haben. Und Lukács' religiös gefärbte Auslegungen Dostoevskijs sind nicht, wie bei Bachtin, bloße Bilder zur Verdeutlichung der künstlerischen Konstruktion. Er sieht in Dostoevskijs Werk vielmehr Anzeichen einer geschichtlichen Wende und einer „zweiten Ethik“, die auf Barmherzigkeit und Liebe gründet. ${ }^{6}$

Dieser Wendepunkt wird in der „Theorie des Romans“ nur angedeutet. Weil es nach Lukács jedoch nur zwei Objektivationen der großen Epik, die Epopöe und den Roman,

4 Zum Zusammenhang und Unterschied zwischen Bachtin und den Formalisten vgl. Freise (1993, 6398) und Tihanov (1998a). Bachtin versteht unter Dialog nicht formalistisch ein bloßes „Verfahren“, sondern ein Mit- und Gegeneinander von „Äußerungen“, in denen die Weltsicht der Sprecher erscheint (Tihanov 1998a, 44f., 47).

5 Weiter als der Herausgeber Nyri geht hier Tihanov. Die Dostoevskij-Notizen nehmen aus seiner Sicht in fragmentarischer Form die Thesen der "Theorie des Romans" vorweg. Lukács habe wenig neue Ideen hinzugefügt (Tihanov 1997, 178).

6 Zu diesem Punkt vgl. das Kapitel „Die zweite Ethik“ bei Bolz (1989, 13-20). Die „Notizen“ zeigen unmißverständlich an, daß Lukács in Dostoevskij nicht den Begrunder der modernen Fiktion und dieselbe als Übel erblickt, wie Corredor meint (1983, 106f.), sondern umgekehrt: eine Erlösung vom Übel. 
gibt (Lukács 1920, 44), liegt der Gedanke nahe, daß Dostoevskijs Werke als neue Epopöen zu verstehen sind. Die Epopöe rückt nicht Individuen, sondern die Gemeinschaft unter einer göttlichen Obhut in ihr Zentrum. Noch gibt es keinen Widerspruch zwischen Seele und Wesen, zwischen der Welt des Ich und der Welt der Taten. Die epische Welt ist nach Lukács nur quantitativ und nicht qualitativ verschieden, Schurken und Helden stehen nebeneinander (ebd., 57). Der quantitativen Valenz der Dinge, ihrem Nebeneinander entspricht auch ihre primär räumliche und nicht zeitliche Organisation in der Epopöe. Eine Entwicklung ist gar nicht nötig, da die Epopöe die Frage, „Wie kann das Leben wesenhaft werden", bereits beantwortet hat. Und so muß der epische Held nicht aktiv das Geschehen bestimmen, den Lebenssinn suchen wie sein romanhafter Nachfolger, sondern er kann ihn passiv hinnehmen. Die Handlung läuft nicht auf ein gesuchtes und erhofftes Ziel hinaus. Die Handlung in der Epopöe äußert sich in Form einer potentiell unendlichen, offenen Abenteuerreihe (ebd., 58f., 128f.). Lukács argumentiert in diesem Punkt zwar nicht konsequent, denn auch im Roman „Don Quixote“ kann der Held, dessen Seele enger ist als die umgebende Wirklichkeit, in Form von Abenteuern mit seiner Umwelt in Kontakt treten. Gerade hier zeigt sich der Abstand von Ich und Tat, der Verlust der transzendentalen Heimat, und nicht die epische Verbundenheit. Diese Romanform tendiert aber - nach Lukács - am stärksten zur Epopöe, weil sie die Zeit als sinnfälligstes Kennzeichen transzendentaler Obdachlosigkeit nicht zu gestalten vermag (ebd., 140).

Auch Bachtin legt den Werken Dostoevskijs ein Abenteuersujet zugrunde, das die Freiheit der Helden von der Zeit, ihr räumliches Nebeneinander, ihre Äquivalenz unterstützt (Bachtin 1994b, 74-80). Das Abenteuersujet hat aus der Sicht Bachtins aber nur dienende Funktion, es ist vor allem kein Indiz des Epischen, und Dostoevskij bleibt für ihn der Autor von Romanen. Während Lukács in der unendlichen Abenteuerreihe ein Zeichen der epischen Totalität erblickt - die Frage ist beantwortet, die Antwort kann beliebig oft gegeben werden - unterstützt das Abenteuersujet nach Bachtin umgekehrt die Unabgeschlossenheit der Helden Dostoevskijs (ebd., 77f.). Die epische Welt braucht nach Lukács keine Architektonik, weder Anfang noch Ende, weil sie in sich geschlossen ist. Bachtin diagnostiziert das Gegenteil: Dostoevskij verwendet das Abenteuersujet, weil er eine offene Welt offener Menschen gestaltet. Mit Hilfe der Abenteuerreihe können Dostoevskijs Helden in außergewöhnliche Situationen gebracht werden, was im biographischen Roman nur schwer gelingt. Die Außergewöhnlichkeit des Umfelds läßt die Persönlichkeit der Helden voraussetzungslos erscheinen. Diese Besonderheit zeigt sich deutlich im zwischenmenschlichen Kontakt. Das Abenteuersujet führt zu unerwarteten Begegnungen von Figuren, zu Überkreuzungen von ideologischen Horizonten, zu Dialogen. Die Interferenz menschlicher Positionen findet laut Bachtin in Dostoevskijs Romanen zum ersten Mal einen adäquaten sprachlichen Ausdruck. Bachtin deutet die stilistisch umgesetzte Vielfalt als Reaktion Dostoevskijs auf das historische Bewußtsein seiner Epoche. Der Pluralismus von Positionen ist Reflex einer sozial desorientierten Intelligenz und Auśdruck eines fehlenden verbindlichen Horizontes (ebd., 158f., 178). Gleichzeitig beugt Bachtin aber auch einer Widerspiegelungsthese vor: Der polyphone Roman entsteht zwar erst mit Dostoevskij, er hat aber Vorläufer in der dialogischen Linie des Romans. In „Problemy tvorčestva Dostoevskogo“ deutet sich bereits die für Bachtins folgende Schriften grundlegende Opposition von monologischem und dialogischem Roman an. Bachtin legt ein $\mathrm{Ne}-$ beneinander und keine dialektische Entwicklung von Gattungen nahe, wobei er der dialo- 
gischen Variante eindeutig den Vorzug gibt. Zumindest implizit unterstellt er diesem Romantyp ein gesellschaftskritisches Potential. Nur die gestaltete Unabgeschlossenheit von Positionen und ihre Vielfalt scheint in der Lage zu sein, einen einheitlichen Horizont etwa ein politisch und kulturell autoritäres Klima - in Frage zu stellen. Bachtin räumt die Möglichkeit ein, daß zu verschiedenen Zeitpunkten der Geschichte - auch in Zukunft auf das Potential des dialogischen Romans einschließlich des Abenteursujets zurückgegriffen werden kann. ${ }^{7}$

Bachtin jedenfalls weigert sich, die „Gemeinschaft von Sündern und Gerechten“" idealistisch zu deuten, wie dies Lukács in seinen Dostoevskij-Notizen zu erkennen gibt. Für Bachtin steht mithin in Dostoevskijs Texten das Nebeneinander disparater Positionen im Vordergrund, während Lukács ganz bestimmte Helden präferieren muß. Es sind dies die epischen „Kinder“, vor allem Fürst Myškin, Sonja Marmeladova und Aleša Karamazov, die um die Einheit ihrer Seele mit dem Wesen wissen und bereits unter dem Schutz der Gottheit stehen, eine „Zweite Ethik“ vertreten. Eine solche Deutung, die aus dem polyphonen Roman einzelne Ideen herausliest, wird von Bachtin kritisiert. Er erwähnt Lukács zwar auch in seiner erweiterten Fassung des Dostoevskij-Buches („Problemy poetiki Dostoevskogo“", 1963) nicht - dies dürfte politische Gründe haben -, einige Ergänzungen können aber als implizite Reaktion auf die „Theorie des Romans“ gedeutet werden. Dazu gehört v. a. Bachtins Tolstoj-Diskussion, die sich klar von Lukács abhebt (1994b, 277ff.). ${ }^{8}$

Tolstojs Romane stellen für Lukács eine Vorstufe der modernen Epopöen Dostoevskijs dar. In Tolstojs Werken liegt noch - wie für den Roman typisch - eine Gesinnung zur Totalität vor, nicht ein Wissen um sie. Diese Gesinnung ist aber bereits mehr als Utopie, mehr als bloße Hoffnung. Tolstojs Helden ahnen bereits etwas von einer neuen Welt, einer neuen Gemeinschaft. Die Ahnung macht sich als Hinwendung zur Natur kenntlich und leuchtet besonders im Angesicht des Todes auf. Den Helden Tolstojs erschließt sich momenthaft ein Weg ,ins lebendige Leben“. Sie sind allerdings im Gegensatz zu Dostoevskijs Helden nicht in der Lage, gemäß dieser Ahnung zu handeln. Eine Rückkehr zur ersten Natur ist keine Lösung, die Menschen fallen notwendig in die Welt der Konvention zurück. Ihre großen Erlebnisse bleiben ohne Folgen. Als Beispiel für die Gestaltung dieses Problems führt Lukács Tolstojs Erzählung „Tri smerti“ an (Lukács 1920, 158; Lukács 1985, 51f.).

7 Den ahistorischen Aspekt in Bachtins Zweilinienmodell akzentuiert Neubauer, womit die Möglichkeit einer Kritik an jedem autorităren Diskurs (auch dem sozialistisch-realistischen) offengehalten werde (Neubauer 1996, 540f.). Tihanov dagegen interpretiert die Opposition von monologischem und dialogischem Roman als historischen Konflikt mit einer intendierten dialogischen ,Lösung'. Er zieht einen Vergleich zur Aufhebung von Verdinglichung und Klassengesellschaft, wie sie Lukács in „Geschichte und Klassenbewußtsein“ vorsieht. Allerdings zeige sich Bachtin 1929 („Problemy tvorcestva Dostoevskogo“) noch unentschieden in der Wertung des Prozesses (Tihanov 1997b, 76f., 87). In einer anderen Studie geht Tihanov jedoch von der "metaphysischen Notwendigkeit" zweier oppositioneller Kräfte (einer zentripetalen, monologisierenden und einer zentrifugalen, dialogisierenden) bei Bachtin aus, die auf jeder geschichtlichen Stufe anzutreffen seien (Tihanov 1998a, 46).

8 Weiter zu nennen sind hier die Ergänzung im 2. Kapitel zu Dostoevskijs Kampf gegen die „Verdinglichung" (овеществление) des Menschen (1994b, 269) oder die Fußnote zur Gattungstheorie, in der sich Bachtin mit dem „Wesen“ der „Epopöe“ auseinandersetzt (348). Neubauer (1996, 532f.) spricht von einer „schweigsamen“ Auseinandersetzung Bachtins mit Lukács. 
Bachtin baut nun seine Charakteristik Tolstojs als eines monologischen Autors genau auf der Diskussion von „Tri smerti“ auf. Seine Kritik zielt auf das fehlende Wissen der drei Sterbenden voneinander: Die Verbindung ihrer Leben findet ausschließlich im Bewußtsein des Autors statt, die drei Wesen befinden sich auch als einzelne ganz in seiner Verfügungsgewalt. Damit weist der Text trotz seiner scheinbaren Vielschichtigkeit nur einen einzigen und dominanten Horizont auf, ein Bewußtsein, das dem Ganzen zugrunde liegt. ${ }^{9}$ Bachtins Beschreibung trifft zwar die Konstruktion der Erzählung, sein impliziter Vorwurf bleibt aber fragwürdig. Die übergeordnete Perspektive Tolstojs läßt sich zweifellos nachweisen. Der Tod der Gräfin, an deren Sarg ein Geistlicher Davids Gesänge liest, wird abschließend wie folgt kommentiert: „Doch verstand sie wenigstens jetzt diese großen Worte?" Das Urteil des Autors über die Heldin - sie hätte schon wesentlich früher diese Worte verstehen müssen - überträgt sich als Anspruch förmlich auf den Leser. Tolstojs moralisches Diktat macht sich bemerkbar, es kann vom Rezipienten als Zudringlichkeit empfunden werden. Auf der anderen Seite läßt sich der Tod, vor allem aber eine Mehrzahl von Toden - wie Bachtin ja selbst in seinen frühen Schriften unterstreicht - nur mittels einer konsequenten Außensicht darstellen. Deshalb richtet Bachtin seine Kritik nicht nur auf die monologische Darstellung, sondern auf die Wahl des Themas selbst. Damit würde aber der Tod als literarisches Motiv unmöglich. Man kann Bachtin hier entgegenhalten, daß Tolstoj sein Thema zumindest adäquat gestaltet: Drei Tode stehen annähernd isoliert nebeneinander, keiner der Sterbenden weiß vom anderen, das Sterben hat keine Folgen für das Leben. Lukács rückt vor allem den ethischen Aspekt der Erzählung, das moralische Problem, das gerade in der Isolation liegt und deshalb auch die Konstruktion bedingt, in den Vordergrund. Bachtin übersieht dies, er verharrt im Dualismus von monologischem und dialogischem Roman und gesteht der ersten Variante per se kein kulturkritisches Potential zu.

Dieser Dualismus wird in dem 1941 entstandenen Aufsatz „Epos und Roman“ vorübergehend nivelliert. Hier spricht Bachtin dem Roman generell kritische Fähigkeiten zu. Es geht ihm - wie bereits der Titel signalisiert - um die offenbar fundamentalere Opposition von Epos und Roman. Das Thema selbst wie auch die Charakterisierung der beiden Gattungen können als Reaktion auf Lukács' „Theorie des Romans" verstanden werden (Neubauer 1996, 542, Tihanov 1998a, 52, Wegner 1987, 78). ${ }^{10}$ Für Bachtin repräsentiert die Epopöe wie für Lukács eine homogene, in sich geschlossene Welt, die eines formalen Anfangs und Endes nicht bedarf (Bachtin 1986, 418). Es gibt keinen Widerspruch zwischen dem epischen Menschen und seinen Taten (ebd., 421), alles ist rund, vollendet, die Zeit absolut. Gleichzeitig verhindert die absolute Vollendung jedoch einen Bezug zur Gegenwart: Was groß ist, das war. Anders als Lukács sieht Bachtin im Epos die absolute Zeit der Vergangenheit gestaltet, von der sowohl Autor als auch Rezipient durch eine unauflösbare epische Distanz geschieden sind (ebd., 401)." Er nähert sich damit seiner frühen Ästhetik an, doch reduziert er seine ursprünglich allgemeine Bestimmung der Kunst nun auf das Epos.

9 Zu Bachtins Verhältnis zu Tolstoj vgl. Emerson (1989, 161).

10 Corredor (1983) und Aucouturier (1983) legen ihren Analysen primär diese beiden Texte zugrunde. Die Reduktion der Position Bachtins auf die Untersuchung „Ëpos i roman“ ungeachtet aller früheren und späteren Abweichungen ergibt allerdings ein einseitiges Bild.

11 Bachtin hält sich dabei an die Definition von Goethe und Schiller, nach der das Epos vollkommene Vergangenheit thematisiert, während Lukács die vollkommene Vergangenheit zur Zeitlosigkeit umdeutet (Neubauer 1996, 533, 542f.). 
Der Roman dagegen ist die einzige künstlerische Form, die in ihrem Werden der Gegenwart entspricht und auf eine sich bildende Welt, eine noch unbestimmte Zukunft reagieren kann. Nur dieses Genre zeigt sich laut Bachtin selbst als unfertig, offen, kritisch und selbstkritisch (ebd., 395f., 399). Damit bezieht Bachtin bestimmte Charakteristika, die er in seinem Dostoevskij-Buch den Helden des dialogischen und polyphonen Romans vorbehalten hatte, jetzt auch auf den Roman generell. Den Beginn der Romanentwicklung legt er in der Antike fest, wo der Roman den Gegenpol zu den kanonischen Gattungen bildet. Dank seiner Flexibilität ist allein der Roman, der sich aus den ,niederen" Sphären der Wirklichkeit mitsamt ihrer Sprachvielfalt speist (ebd., 408), in der Lage, geschichtlichen Veränderungen zu folgen. Er löst deshalb das Epos als dominantes Genre ab. Je dringender die Probleme der Gegenwart auf die Kunst zurückwirken, desto größer wird die Vormachtstellung des Romans, die sich besonders deutlich seit der zweiten Hälfte des 19. Jahrhunderts beobachten läßt. Bachtin schließt jedoch ein Wiederaufleben des Epischen nicht aus. Seine Einschätzung von Gogol's „Mertvye duši“ als intendierte Epopöe, die zum Roman geriet, gleicht derjenigen Lukács' auffallend (Bachtin 1986, 415, Lukács 1920, 109). Allerdings traut Bachtin im Unterschied zu Lukács modernen Epopöen nicht die Fähigkeit zu, ein Ideal für die Gegenwart zu vermitteln. Die Funktion des Epos besteht laut Bachtin in der positiven Gestaltung der nationalen Vergangenheit (Bachtin 1986, 401). Dostoevskijs Romane leisten dies auf alle Fälle nicht. Bachtin setzt sich damit deutlich von Lukács' Dostoevskij-Idealisierung ab; er ist aber seinerseits nicht frei von einer Idealisierung des Romans. ${ }^{12}$

\section{Die Differenzierung der Romanzeit: Der Chronotopos als zentrale Unterscheidungska- tegorie}

Lukács mißt der Zeitkonzeption in Epos und Roman signalhafte Bedeutung zu: Die gegensätzlichen Weltbilder dieser beiden Gattungen ziehen verschiedene Zeitkonzeptionen nach sich. Von zentraler Bedeutung ist dabei die Tatsache, daß Lukács die Zeit als abhängige Variable begreift: Jede Kulturepoche erschafft sich ihr eigenes Zeitgefühl. Aus Lukács Sicht ist das antike Epos deutlich zeitenthoben, die Abenteuer der antiken Helden spielen sich in einer vektorlosen kosmischen Zeit $\mathrm{ab}$, in der es weder individuelle Lernprozesse noch historische Evolutionen geben kann. Sogar das biologische Alter erscheint als unveränderliche Eigenschaft: „Nestor ist alt, so wie Helena schön und Agamemnon mächtig ist." (Lukács 1920, 128) Lukács stellt fest, daß die Zeit im Epos parallel zu räumlichen Vorstellungen strukturiert ist - die Figuren können sich in jeder Richtung frei bewegen (ebd., 128). Für die Charakterisierung der Zeit im modernen Roman hingegen greift

12 In der zunehmenden Beeinflussung aller kanonischen Gattungen durch den Roman sieht Tihanov eine Analogie zur Aufhebung der Klassengegensätze mit dem Ziel einer klassenlosen Gesellschaft bei Lukács („Geschichte und Klassenbewußtsein“). Die Gattung des Romans entspricht dabei der Klasse des Proletariats. Den Unterschied beider Theorien macht Tihanov an der Art des intendierten Umschwungs fest: Die dialogische Veränderung steht der Revolution gegenüber (Tihanov 1997b, 86). Daneben zeigt Tihanov aber Bachtins Unentschiedenheit zwischen einer historischen und metaphysischen Argumentation. Bachtin gehe zum einen von zwei ahistorischen und konträren Weltsichten in Epos und Roman aus, zum anderen postuliere er - ähnlich Lukács - eine geschichtliche Entwicklung mit einem - inhaltlich von Lukács abweichenden - utopischen Ziel: Erhofft wird die Dominanz des Romans (Tihanov 1998a, 51-55). 
Lukács auf die Bergsonsche Kategorie der durée zurück: Die fließende Zeit verwandelt sich im individuellen Erleben in ein Kontinuum, das sich der künstlerischen Schau erschließt. Lukács konzentriert seine Ausführungen auf den Helden, der im Zentrum seiner Romantheorie steht. Zeit im Roman ist für Lukács immer die subjektive Zeit des Helden, der nicht mehr über die epische und zeitaufhebende „Lebensimmanenz des Sinnes“ verfuigt. Mehr noch: Die Zeit als solche, die Biographien, Prozesse und Entwicklungen erst ermöglicht, ist konstitutiv für das Romangenre:

Nur im Roman, dessen Stoff das Suchenmüssen und Nicht-finden-Können des Wesens ausmacht, ist die Zeit mit der Form mitgesetzt [...]. Im Roman trennen sich Sinn und Leben und damit das Wesenhafte und Zeitliche; man kann fast sagen: die ganze innere Handlung des Romans ist nichts als ein Kampf gegen die Macht der Zeit. $(1920,129)$

Lukács entwirft ein binäres Modell: Der Zeitenthobenheit des antiken Epos steht die subjektive Romanzeit gegenüber. Zwischen diesen beiden Polen spielt sich seine ganze Argumentation ab.

Bachtins Ausführungen zu diesem Problemkreis unterscheiden sich in dreifacher Hinsicht von Lukács' Überlegungen. Erstens erweitert Bachtin die Analyse der Romanzeit um die Kategorie des Raumes und definiert seinen zentralen Untersuchungsgegenstand, den Chronotopos, als „wesenhafte Wechselwirkung zwischen zeitlichen und räumlichen $\mathrm{Be}-$ ziehungen, wie sie sich in der Literatur künstlerisch ereignen" (Bachtin 1986, 121). Zweitens statuiert er keine historische Entwicklung von antiker Zeitlosigkeit im Epos zum modernen Zeitbewußtsein im Roman; aus Bachtins Sicht koexistieren mehrere Chronotopoi von Anfang an nebeneinander. Und drittens beschränkt sich Bachtin nicht auf die subjektive Zeit des Romanhelden, sondern rechnet auch mit der Möglichkeit einer kollektiven Zeit, die sich besonders deutlich im folkloristischen Chronotopos äußert (Dembski 1998, 197f.).

Obwohl Bachtin keine geschichtsphilosophische Gegenüberstellung von Epos und Roman anvisiert, versucht er doch, eine literarhistorische Ordnung der verschiedenen Romanchronotopoi vorzunehmen. In seiner Untersuchung „Formy vremeni i chronotopa v romane“ beschreibt Bachtin die Grundtypen, die nicht in allen Kulturepochen in gleichem Maße präsent sind, aber nach ihrem erstmaligen Auftreten immer wieder aktualisiert werden können: Die Abenteuerzeit des griechischen Romans, die biographische Zeit des Bildungsromans, die Folklorezeit des karnevalistischen Romans, die idyllische Zeit des Heimatromans u. a. Für jeden Zeittypus arbeitet Bachtin die zentralen Bedeutungskategorien heraus: Die Abenteuerzeit operiert mit dem „Plötzlichen", die biographische Zeit mit der „Entwicklung“, die Folklorezeit mit dem „magisch-rituellen Zyklus“. Diese Zeitbegriffe determinieren in Bachtins Deutung die Romanhandlung, mehr noch: Sie stecken den Rahmen desjenigen ab, was in der dargestellten Welt überhaupt möglich ist.

Ähnliches gilt auch für den Raum, der die zeitliche Organisation komplementiert. Die Abenteuerzeit präferiert den „Weg“ als Handlungsort, hier ereignen sich „plötzliche“ Begegnungen am leichtesten, andere Zeitformen gehen Hand in Hand mit anderen Räumen, so z. B. der „Provinzstadt“, dem „Haus“ usw. Die Aufzählung der einzelnen Chronotopoi dient Bachtin zur Erstellung einer Taxonomie der verschiedenen Romantypen. Dabei eignet sich gerade der Chronotopos am besten als zentrales Unterscheidungskriterium, weil er 
die Romanwirklichkeit konstituiert. Außerhalb des Chronotopos ist gar keine literarische Darstellung denkbar. ${ }^{13}$

Der entscheidende Unterschied zwischen Bachtin und Lukács liegt in der Differenzierung des Abhängigkeitsverhältnisses von künstlerischer Zeitkonzeption und Epochenbewußtsein. Bei Lukács ist das biographische Zeitbewußtsein des Romans deutlich durch das "Zeitalter vollendeter Sündhaftigkeit" bedingt. Hieraus ergibt sich die geschichtsphilosophische Dimension der Romanzeit. Auch Bachtin erklärt die Entstehung der Chronotopoi aus ihrem historischen Umfeld, der folkloristische Chronotopos entspricht z. B. den Lebensbedingungen der Ackerbaugesellschaft. Allerdings gilt dieser historische Bezug nur für die Genese eines Chronotopos. Nachdem sie eine bestimmte Epoche dominiert haben, leben bereits bestehende Chronotopoi gewissermaßen in einer Art Ruhezustand weiter und können auch in einem anderen historischen Kontext aktiviert werden. Bachtin geht von der Wahlmöglichkeit des Autors aus und setzt ein Spektrum verschiedener Chronotopoi bereits in der Antike an.

Bachtins radikale Modifikation von Lukács' Modell äußert sich am deutlichsten durch die Einführung eines neuen Genres. Immer wieder spricht Bachtin vom ,griechischen Roman", während für Lukács in der Antike nur Epen denkbar sind. Für Lukács ist der Roman ein Krisenphänomen, das dem Verlust des antiken Sinns für Totalität entspringt. Die antike Welt kennt noch das Allumfassende, Homogene, Abgeschlossene. Jede Einzelerscheinung hat im Kosmos der Dinge seinen Ort, und das Epos stellt diese Totalität literarisch dar. Bachtin hingegen setzt den Zerfall der einheitlichen Weltsicht schon in der Antike an und wertet dieses Phänomen darüber hinaus positiv. Er unterstreicht insbesondere die Wichtigkeit der komischen Genres (v. a. der menippeischen Satire) für die Entwicklung des Romans.

Jede literarische Darstellung ist für Bachtin partiell, weil sie durch den gewählten Chronotopos eingeschränkt wird. Das gilt in gleichem Maß für das Epos wie für den Roman. Bachtin stellt damit die von Lukács behauptete kosmische Totalität des Epos in Frage. Das Epos zeichnet sich zwar auch für Bachtin durch Abgeschlossenheit aus, diese impliziert aber gleichzeitig Unzugänglichkeit. Epen können nur lange post factum entstehen. Die Zeit ist immer eine erinnerte. Bachtin hebt die absolute Distanz des episch Beschriebenen zur Gegenwart des Schreibenden hervor. Das Epos repräsentiert mithin nicht kosmische Totalität, sondern nur statische, fernliegende Geschlossenheit $(1984,405)$.

Schließlich ist der Chronotopos für Bachtin in erster Linie eine textuelle Kategorie, die die Zeitrepräsentation im literarischen Kunstwerk regelt. Lukács hingegen richtet seine Aufmerksamkeit ausschließlich auf die subjektive Zeiterfahrung des Romanhelden; die allgemeine zeitliche Organisation des Textganzen bleibt außerhalb seines Erkenntnisinteresses. Eine Gemeinsamkeit von Lukács' und Bachtins Romantheorien liegt aber in ihrem literaturgeschichtlichen Interesse. Lukács operiert deutlich vor dem Hintergrund einer dialektischen Geschichtsphilosophie, die das Epos als These, den Roman als Antithese und eine neue Epopöe, die sich im Werk Dostoevskijs ankündigt, als Synthese begreift. Bachtin

13 Die Nähe zu Kant ist unverkennbar. Bachtin nennt den Namen Kants in einer Fußnote, bemerkt aber einschränkend, daß Raum und Zeit in seinem Verständnis nicht transzendentale Formen der Erkenntnis seien, sondern Formen der realen Wirklichkeit selbst $(1986,122)$. In den 1973 entstandenen „Schlußbemerkungen“ gibt Bachtin diese Position jedoch wieder auf und erklärt, daß die „Sphäre der Sinnbildungen nur durch die Pforte der Chronotopoi betreten“ werden könne (290). 
hingegen rechnet mit einer mehrgleisigen Entwicklung. Dabei bevorzugen die einzelnen kulturhistorischen Epochen bestimmte Chronotopoi. Allerdings kann nicht jeder Chronotopos ausschließlich einer Epoche zugeschrieben werden, Bachtin sieht explizit Mehrfachzuordnungen und Mischformen vor. ${ }^{14}$

\section{Die Dezentralisation des Bewußtseins: Ironie vs. Karneval}

Von allen Konzepten Bachtins hat vermutlich die „Karnevalisierung“ in der westlichen Literaturwissenschaft die intensivste Rezeption erfahren (Morson; Emerson 1990, 4). Bachtin definiert den Karneval als Neuordnung des Lebens und weist vor allem auf seine Ventilfunktion für die Menschen hin, die in Regelkulturen leben. Der Karneval verwandelt das Leben in ein fröhliches Theaterstück; er inszeniert eine von allen Zwängen befreite Gegenwelt: „Im Karneval spielt das Leben selbst, indem es [...] eine andere freie (selbständige) Form seiner Verwirklichung erprobt, seine Wiedergeburt und Erneuerung nach besseren Prinzipien." (Bachtin 1986, 298)

In dieser Formulierung scheint der romantische Kern von Bachtins Karnevalsbegriff auf. Bereits die Romantik versuchte nämlich ein philosophisches Konzept aufzufinden, das die Bedingtheit der menschlichen Existenz erträglich machen sollte. Das zentrale Prinzip, das zwischen Endlichem und Unendlichem vermittelt, heißt bei den Romantikern zwar ,Ironie', es verfügt aber über alle jene Merkmale, die Bachtin später als konstitutiv für den Karneval aufzählen wird: Im ironischen Sprechen erkennt der Mensch die Grenzen seines Daseins, nimmt aber gleichzeitig eine Außenposition ein, von der aus er seine conditio humana inspizieren kann.

Lukács greift den romantischen Ironiebegriff unmittelbar auf und verwendet ihn in seiner Romantheorie. Die Ironie im Roman stellt bei Lukács ein Surrogat für die verlorene epische Totalität dar: „Die Ironie des Dichters ist die negative Mystik der gottlosen Zeiten: eine docta ignorantia dem Sinn gegenüber." (Lukács 1920, 87f.) An prominenter Stelle seiner „Theorie des Romans“, nämlich am Ende des ersten Teils, weist Lukács noch einmal mit allem Nachdruck auf die zentrale Rolle der Ironie bei der Ausbildung des modernen Romans hin:

Die Ironie als Selbstaufhebung der zu Ende gegangenen Subjektivität ist die höchste Freiheit, die in einer Welt ohne Gott möglich ist. Darum ist sie nicht bloß die einzig mögliche apriorische Bedingung einer wahrhaften, Totalität schaffenden Objektivität, sondern erhebt auch diese Totalität, den Roman, zur repräsentativsten Form des Zeitalters, indem die Aufbaukategorien des Romans auf den Stand der Welt konstitutiv auftreffen. (ebd., 91)

Noch 1949, nach der Wende zum Marxismus, wird Lukács sein romantisches Ironiekonzept mit Puskins „Roman in Versen“ „Evgenij Onegin“ in Verbindung bringen. Auch hier schreibt Lukács die Schaffung von Totalität der spielerisch-ironischen Diktion des Erzäh-

14 Bachtin läßt allerdings die Frage offen, ob bestimmte Chronotopoi den von ihm beschriebenen parallelen Romanlinien, der monologischen und der dialogischen, zuzuordnen wären: ,[...] the two main vectors in Bakhtin's theory of the novel - the classification based on chronotope and the typology that rests on the opposition between monologue and dialogue/polyphony - never intersect." (Tihanov $1998 \mathrm{~b}, 123$ ) 
lers zu; die Konzession an die Ästhetik des sozialistischen Realismus besteht in der Schlußfolgerung, daß eine solche Darstellung „der treuen Widerspiegelung der Wirklichkeit des Lebens Schönheit verleiht" (Lukács 1969, 25f.).

In der "Theorie des Romans“" leitet Lukács seine Romantypologie mit Überlegungen zu „Don Quixote“ ein, den er in den Rang des ,ersten großen Roman[s] der Weltliteratur“ erhebt (Lukács 1920, 104). Besondere Bedeutung mißt Lukács der Tatsache bei, daß „Don Quixote" ursprünglich als Parodie auf die geschichtlich überholte Form der Ritterromane konzipiert wurde. In seinem Roman habe Cervantes intuitiv das problematische Gegenüber eines idealistischen Helden und einer bereits toten transzendentalen Ordnung gestaltet - deshalb präsentiere sich „Don Quixote“ als ein „Ineinander von Poesie und Ironie, von Erhabenheit und Groteske, von Göttlichkeit und Monomanie“ (ebd., 105f.).

Lukács richtet hier sein Augenmerk auf eine Kategorie, die ihn leicht auf eine Bachtinsche Argumentationslinie hätte führen können. Bachtin erblickt nämlich in der Parodie den ersten Keim des dialogischen Prinzips: In parodistischen Texten überlagern sich der parodierte und der parodierende Diskurs - das Wort wird somit zweistimmig. In den Ausführungen zu den ",beiden stilistischen Linien des europäischen Romans" wird bereits in der Wortwahl deutlich, daß Bachtin in seiner Genealogie des polyphonen Romans auf Lukács' "Theorie des Romans" rekurriert, allerdings mit einer entscheidenden Veränderung: „Der Roman setzt die verbal-semantische Dezentralisierung der ideologischen Welt voraus, die sprachliche Obdachlosigkeit eines literarischen Bewußtseins" (Bachtin 1975, 178). Während Lukács auf der transzendentalen Obdachlosigkeit des Individuums beharrt, betont Bachtin die sprachliche Obdachlosigkeit des literarischen Bewußtseins. Bachtin verlegt also Lukács' geschichtsphilosophische Problematik in den Bereich der Literarizität. Bei aller Gegensätzlichkeit, die das Verhältnis Bachtins zu den Formalisten auszeichnet, kann man bei Bachtin in diesem Fall eine formalistische Denkfigur diagnostizieren: Die Erklärung des literarischen Prozesses ausschließlich durch innerliterarische Faktoren gehört zu den Basispostulaten der Formalisten (Tihanov 1997c, 281).

Im seinem „Satire“-Artikel, der für die „Literaturnaja enciklopedija“ bestimmt war, weist Bachtin auf den Zusammenhang zwischen Parodie und Roman nur in einer Klammer hin in aller Kürze wird hier aber das Wesentliche gesagt: ,[der Roman] wurde durch die Satire vorbereitet und erneuerte sich in der Folge mit Hilfe des satirischen und parodistischen Elements." (Bachtin 1996, 13)

Während die Ironie bei Lukács das Verhältnis zwischen Seele des Romanhelden und der Wirklichkeit bezeichnet, tritt sie bei Bachtin primär als Beziehung zwischen Texten auf. ${ }^{15}$ In einem parodistischen Text wird ein bereits bestehender Diskurs von einem zweiten Diskurs herausgefordert - dieser innere Widerstreit der Aussagemodi repräsentiert für Bachtin das wesentliche Grundprinzip des modernen polyphonen Romans.

Bachtin blendet aber Lukács' Problem nicht einfach aus. Auch für ihn kommt das Verhältnis des menschlichen Bewußtseins zur äußeren Wirklichkeit in den Blick. Allerdings

15 Zwar bespricht Bachtin in seinem Lexikonartikel auch die satirische Negation der Wirklichkeit, verschiebt dann aber seine Argumentation sofort auf die Beziehung zwischen Texten: „Das satirische Moment trägt in jedes beliebige Genre ein Korrektiv der zeitgenössischen Wirklichkeit, der lebendigen Aktualität, politischer oder ideologischer Mißstände hinein. Das satirische Element, das gewöhnlich untrennbar mit der Parodie oder der Travestie verbunden ist, reinigt jedes Genre von erstarrender Bedingtheit, von sinnlos und überflüssig gewordenen traditionellen Elementen." (1996, 12f.) 
dreht Bachtin hier die Kausalität um. Für Lukács besteht die romanhafte Ironie der menschlichen Seele darin, daß sie sich an eine starre, gottlose Welt anpassen muß. Bachtin sieht umgekehrt in seinem Karnevalskonzept die Möglichkeit vor, daß sich die Welt zumindest zeitweise nach den Wünschen des Menschen richtet. Mit anderen Worten: Bei Lukács ermöglicht die romanhafte Ironie die seelische Bewältigung der sinnentleerten Wirklichkeit, Bachtin hingegen erblickt im Karneval eine ironisch idealisierte Wirklichkeit.

Dabei richtet Bachtin seine besondere Aufmerksamkeit auf die Schnittstelle zwischen Innen- und Außenwelt des Menschen: auf seinen Körper. Es ist kein Zufall, daß Bachtin als Kronzeugen für den modernen Roman nicht Cervantes, sondern Rabelais auswählt. In "Don Quixote" spielen körperliche Motive fast keine Rolle; am Beispiel dieses Romans belegt Bachtin wiederholt seine These, daß der dialogische Roman sich aus der literarischen Parodie entwickelt hat. Nie aber taucht Cervantes im Zusammenhang mit dem Karnevalskonzept auf. „Don Quixote“ bildet in Bachtins Interpretation eine Vorstufe zu „Gargantua et Pantagruel“. Während sich das prekäre Verhältnis von Cervantes' Protagonisten zur Wirklichkeit auf ein intellektuelles Nichtverstehen, ein diskursives Abseitsstehen beschränkt, setzt Rabelais das Fremdsein seiner Hauptfiguren auch figural um: Die Grenzen zwischen Körper und Welt werden neu gezogen. Es scheint, daß Bachtin gerade in seiner Behandlung von Rabelais' Narren auf Lukács' Konzept der „transzendentalen Obdachlosigkeit" zurückgreift. Immer wieder setzt Bachtin in seiner Diskussion dieser Figur die Adjektive ,fremd“", „unbehaust", „unverwurzelt" ein (Morson; Emerson 1990, 352). Gerade weil der Narr nicht „von dieser Welt“ ist und „nicht versteht“ (Bachtin 1986, 195), ist er ein Romanheld par excellence. Allerdings geht Bachtins Begründung in eine neue Richtung: Während für Lukács die Defiziterfahrung des „obdachlosen“ Romanhelden im Vordergrund steht, argumentiert Bachtin diskurstheoretisch. Die für den Roman konstitutive Vervielfachung der Diskurse ist für Bachtin bereits in der Rolle des Narren angelegt; sein „Nichtverstehen“ macht sich neben dem traditionellen „Verstehen“ als zweite Stimme bemerkbar. Bachtin weist in diesem Zusammenhang auf die Nähe des Narren zur Figur des „Sonderlings“ hin, der so oft im Zentrum der Romane des 18./19. Jahrhunderts steht (Sterne, Jean Paul, Dickens u. a.). Der romanhafte „Sonderling" muß allerdings bereits als Spätform des Narren begriffen werden; die Entwicklung dieser Figur gipfelt für Bachtin in Rabelais' karnevalistischer Körperkonzeption, die sich etwa noch in Gogol's Erzählung „Nos" deutlich bemerkbar macht.

\section{Zusammenfassung}

In den Romantheorien von Lukács und Bachtin zeigen sich grundlegende Gemeinsamkeiten und tiefgreifende Unterschiede. Beide Denker gehen in ihren frühen Schriften von einem impliziten ethischen Standpunkt aus und sprechen der Kunst eine Orientierungsfunktion im Leben zu. Sowohl für Lukács als auch für Bachtin weist das Kunstwerk über sich selbst hinaus, weil das Subjekt (der Autor) hier ein Objekt (den Helden) vollenden kann und so auf eine Norm verweist. Später sind die thematischen Übereinstimmungen zwischen Lukács und Bachtin noch offensichtlicher: Im Zentrum ihres Interesses steht der Roman und seine Entwicklung bis hin zu Dostoevskij. Während aber Lukács weiterhin dem Ideal der Vollendung verpflichtet bleibt und es in eine dialektische Geschichtsphilosophie einschreibt, kehrt Bachtin seine frühe Position um: Lukács deutet Dostoevskijs 
Romane als moderne Epopöen, Bachtin versteht sie als polyphone offene Romane. Beide teilen die Einschätzung, daß in Dostoevskijs Welt Geschichte, darunter die Biographie der Helden, keine Rolle spielt. Dieses Ungeschichtliche erfährt aber eine konträre Auslegung: Lukács sieht darin das Vorzeichen einer neuen Epoche, in der Totalität wieder erscheinen kann. Bachtin hingegen akzentuiert das Offene der Gegenwart, das in einem Pluralismus menschlicher Positionen, in einer stilistisch realisierten Vielstimmigkeit zum Ausdruck kommt. Historisch gesehen postuliert Bachtin zwei parallel verlaufende Romanlinien, eine monologische (Tolstoj) und eine dialogische (Dostoevskij). Damit stellt er Lukács' eingleisiger Entwicklung ein binäres literaturgeschichtliches Modell entgegen, das alternative Weltdeutungen zuläßt.

Nach 1940 zeigt sich Bachtins Bezugnahme auf Lukács deutlich in der Gegenüberstellung von Epos und Roman. Die von Lukács beschriebene historische Ablösung des Epos durch den Roman modifiziert Bachtin, indem er bereits in der Antike eine Opposition konträrer künstlerischer Weltsichten annimmt. Für Bachtin thematisiert das Epos absolute Vergangenheit, während der Roman in offener Form auf die Gegenwart reagiert. Eine Renaissance von Epopöen in der Gegenwart ist damit nicht ausgeschlossen, könnte nach Bachtin jedoch nur die Idealisierung des Vergangenen implizieren und nicht wie bei Lukács eine geschichtliche Wende andeuten.

Bachtin unterteilt die einzelnen Romanformen weiter anhand von Zeit-Raum-Konzeptionen (Chronotopoi). Damit differenziert er Lukács' Gegenüberstellung von räumlichem Zeitverständnis im antiken Epos und eindimensional ausgedehnter Zeit im Roman. Für Bachtin konstituiert sich ein Chronotopos zwar nicht unabhängig von der geschichtlichen Epoche, er lebt aber im literarischen Bewußtsein weiter und kann je nach geschichtlicher Situation reaktiviert werden. Es ist davon auszugehen, daß ein Autor immer mehrere Möglichkeiten der Zeitdarstellung und damit der Weltdeutung hat.

Bachtins Theorie ist nicht wertfrei: Er präferiert die dialogische Romanlinie und den Folklore-Chronotopos. Aus dieser Besonderheit läßt sich sein Interesse für den Karneval erklären. Es ist gleichzeitig möglich, Bachtins subversives Karnevalskonzept als Weiterführung von Lukács' romantischem Ironie-Begriff zu deuten. Die Ironie im Roman, für Lukács Zeichen der „Gesinnung zur Totalität“ im Zeitalter transzendentaler Obdachlosigkeit, wird von Bachtin literarisch und immanent gefaßt: Die Ironie setzt eine zweite Stimme voraus, die eine bereits vorhandene Stimme parodiert. Deshalb steckt in der Parodie und in der Satire der Kern zum polyphonen Roman.

Zusammenfassend läßt sich festhalten, daß Bachtin zwar vielfältig auf Lukács' Deutungskategorien zurückgreift, diese aber in einen neuen theoretischen Gesamtzusammenhang bringt. Bachtins Romantheorie kann damit als diskursanalytisches Gegenstück zu Lukács' geschichtsphilosophisch geprägter Literaturkonzeption verstanden werden.

\section{Literatur}

M. AUCOUTURIER (1983), The Theory of the Novel in Russia in the 1930s: Lukács and Bakhtin, in: The Russian Novel from Pushkin to Pasternak. Hrsg. von J. GARRARD. New Haven, 229-240.

M. BACHTIN (1975), Voprosy literatury i èstetiki: Issledovanija raznych let. M.

M. BACHTIN (1986), Literaturno-kritičeskie stat'i. M.

M. BACHTIN (1994a), Raboty 20-ch godov. Kiev.

M. BACHTIN (1994b), Problemy tvorčestva Dostoevskogo. Kiev. 
M. BACHTIN (1996), Sobranie sočinenij. Tom 5: Raboty 1940-ch - načala 1960-ch godov. M.

N. BOLZ (1989), Auszug aus der entzauberten Welt: Philosophischer Extremismus zwischen den Weltkriegen. München.

K. CLARK; M. HOLQUIST (1984), Mikhail Bakhtin. Cambridge; London.

E. CORREDOR (1983), Lukács and Bachtin: A Dialogue on Fiction, in: Revue de l'Université d'Ottawa/University of Ottawa Quarterly 53, 97-107.

T. DEMBSKI (1998), Lukács, Bachtin und Rilke: Paradigmen der Romantheorie zu Beginn des 20. Jahrhunderts. Diss. Berlin.

C. EMERSON (1989), The Tolstoy Connection in Bakthin, in: Rethinking Bakthin: Extensions and Challenges. Hrsg. von G. S. MORSON und C. EMERSON. Evanston, 149-170.

M. FREISE (1993), Michail Bachtins philosophische Ästhetik der Literatur. Frankfurt am Main. (Slavische Literaturen; 4)

A. KOVÁCS (1980), On the Methodology of the Theory of the Novel: Bachtin, Lukács, Pospelov, in: Studia Slavica Academiae Hungaricae 26, 377-393.

G. LUKÁCS (1911), Die Seele und die Formen. Berlin.

G. LUKÁCS (1920), Die Theorie des Romans: Ein geschichtsphilosophischer Versuch uber die Formen der großen Epik. Berlin.

G. LUKÁCS (1969), Russische Revolution, russische Literatur. Neuwied.

G. LUKÁCS (1974), Die Subjekt-Objekt-Beziehung in der Ästhetik, in: G. LUKÁCS, Heidelberger Ästhetik (1916-1918). Darmstadt; Neuwied, 91-132. (Frühe Schriften zur Ästhetik 2. Werke 17)

G. LUKÁCS (1985), Dostojewski: Notizen und Entwurfe. Budapest.

G. S. MORSON; C. EMERSON (1990), Mikhail Bakhtin: Creation of a Prosaics. Stanford.

J. NEUBAUER (1996), Bakhtin versus Lukács: Inscriptions of Homelessness in Theories of the Novel, in: Poetics Todoy 17, 531-546.

G. TIHANOV (1997a), Bakhtin and Lukács: The Theory of the Novel as Social Philosophy. Diss. Oxford.

G. TIHANOV (1997b), Reification and Dialogue: Aspects of the Theory of Culture and Society in Bakhtin and Lukács, in: Bahtin in humanistihne vede: Zbornik prispevkov $\mathrm{z}$ mednarodnega simpozija $\mathrm{v}$ Ljubljani, 19.-21. oktober 1995; Bakhtin and the Humanities: Proceedings of the Conference in Ljubljana, October, 19-21, 1995. Ljubljana, 73-93.

G. TIHANOV (1997c), Bakhtin, Lukács and German Romanticism: The Case of Epic and Irony, in: Face to Face: Bakhtin in Russia and the West. Hrsg. von CAROL ADLAM u. a. Sheffield, 273-298.

G. TIHANOV (1998a), Bakhtin's Essays on the Novel (1935-41): A Study of their Intellectual Background and Innovativeness, in: Dialogism 1, 30-56.

G. TIHANOV (1998b), The Ideology of Bildung: Lukács and Bakhtin as Readers of Goethe, in: Oxford German Studies 27, 103-140.

M. WEGNER (1987), Romantheorie im Disput: Georg Lukács und Michail Bachtin, in: Socialni realizem v slovenskem jeziku, književnosti in kulturi. Hrsg. von FRANC ZADRAVEC. Ljubljana, 75-81. (Obdobja; 7)

Dr. Andrea Zink, PD Dr. Ulrich Schmid, Slavisches Seminar - Universität Basel, Nadelberg 4, Engelhof, CH-4051 Basel 